\title{
"La paradoja de la movilidad y la ciudadanía": domicialización y documentación tras la deportación en Tijuana
}

\author{
"The paradox of mobility and citizenship": domicilization \\ and documentation after deportation in Tijuana
}

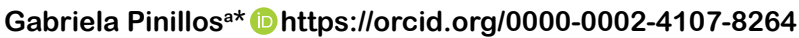

a El Colegio de México. Ciudad de México, México, correo electrónico: gabrielapinillos@gmail.com

\section{Resumen}

El objetivo del artículo es analizar la interacción de la condición de documentación con el acceso a vivienda y la movilidad urbana de personas que viven en Tijuana, México, después de la deportación desde Estados Unidos. Con un enfoque inductivo, entrevistas semiestructuradas a 17 funcionarios y activistas, y con la entrevista de corte biográfico, se realizó un análisis a profundidad sustentado en 23 casos. Los resultados muestran que la visión domiciliada del ciudadano en México responde a la de una ciudadanía fija espacialmente, es decir,

Recibido el 16 de mayo de 2020. Aceptado el 5 de agosto de 2020.

Publicado el 13 de agosto de 2020.

${ }^{*}$ Autora para correspondencia: Gabriela Pinillos. Correo electrónico:

gabrielapinillos@gmail.com

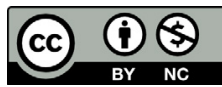

Esta obra está protegida bajo una Licencia Creative Commons Atribución-NoComercial 4.0 Internacional. el acceso a vivienda fija requiere la posesión de documentos de identidad y viceversa. Quienes logran acceder a dichos documentos tienen mayor facilidad para rentar una vivienda, mientras que quienes no lo logran dependen más de la oferta de albergues y se mantienen en constante movimiento intraurbano lo que, a su vez, los somete a una permanente vigilancia y control de la policía.

Palabras clave: documentación, domicialización, reciudadanización, deportación, frontera.

\begin{abstract}
The goal of this article is to analyze the interaction of the documentation status with access to housing and urban mobility of subjects deported from the United States living in Tijuana, Mexico. Through an inductive approach with $17 \mathrm{semi}$ structured interviews with public officials and activists and biographical interviews, an in-depth analysis was carried out based on 23 cases. The results show that the domiciled vision of the citizen in Mexico responds to that of a spatially fixed citizenship, that is, access to fixed housing requires the possession of identity documents and vice versa. Those who manage to access said documents have an easier time renting a home, while those who do not manage to have documents depend more on the availability of shelters and remain in constant intra-urban movement, which, in turn, subjects them to permanent surveillance and control by the police.
\end{abstract}

CÓMO CITAR: Pinillos, G. (2020). "La paradoja de la movilidad y la ciudadanía": domicialización y documentación tras la deportación en Tijuana. ["The paradox of mobility and citizenship": domicilization and documentation after deportation in Tijuana]. Estudios Fronterizos, 21, e052. https://doi.org/10.21670/ref.2010052 
Keywords: documentation, domicilization, re-citizenship, deportation, border.

\section{Introducción ${ }^{1}$}

Las dinámicas y prácticas de la población deportada desde el interior de Estados Unidos que se construyen a lo largo del proceso postdeportación, vinculadas principalmente con el desplazamiento cotidiano en la ciudad de Tijuana en la frontera norte de México, ponen de manifiesto lo que podría denominarse "la paradoja de la movilidad y la ciudadanía”. Una paradoja en la que los individuos, luego de ser deportados a su país de origen y con la dificultad de acceder a documentos de identidad que acrediten su ciudadanía, se mantienen sin domicilio fijo y en constante movimiento diariamente en la ciudad, convirtiéndose en sujetos de vigilancia permanente por el Estado de origen.

En el destino, ${ }^{2}$ los sujetos migrantes entran en una búsqueda por asentarse, tener un domicilio, trabajar y hacer uso del espacio público, pero sus posibilidades están limitadas por la condición de documentación en la que se encuentren (De Génova, 2002; Menjívar, 2006). Esa misma condición parece que continúa luego de la deportación, cuando los sujetos, fundamentalmente sin documentos mexicanos, al momento de la repatriación ${ }^{3}$ y con dificultades para acceder a ellos en sus intentos de reinserción, se mantienen en una permanente itinerancia intraurbana.

La itinerancia intraurbana se plantea como la forma que toma el desplazamiento cotidiano que llevan a cabo en Tijuana aquellos sujetos deportados que se mueven en lo que podría parecer una ruta diaria basada en la alternancia entre lugares de alojamiento, espacios de trabajos por destajo o jornal, espacios públicos de concentración por servicios asistenciales y de reunión por ocio y, a partir de estos, el encuentro con la fuerza pública (Pinillos, 2018). Dicha itinerancia está asociada con la dificultad de tener una vivienda fija y a la ausencia o dificultad de acceder a documentos de identidad (en México el documento que se ha convertido en el requisito para comprobar la identidad es la credencial electoral, llamada comúnmente INE, por las siglas del Instituto Nacional Electoral). Rentar una vivienda requiere contar con documentos de identidad y, a su vez, acceder a estos requiere de la presentación de un comprobante de domicilio fijo.

El objetivo de este artículo, por tanto, es mostrar y analizar la interacción de la condición de documentación con el acceso a vivienda y la movilidad urbana como parte del proceso de reinserción social de personas que viven en Tijuana, México,

\footnotetext{
${ }^{1}$ Los hallazgos presentados en este artículo forman parte de la investigación de tesis doctoral denominada "Volver a ser mexicano: reciudanización y deportación en la frontera México-Estados Unidos".

${ }^{2}$ La observación de los flujos migratorios a nivel mundial y las nuevas perspectivas teóricas vinculadas a la migración, dan cuenta del carácter circular de la movilidad humana y derrumban el mito de la migración como un proceso lineal con un punto inicial y un punto final, por lo que los términos destino, retorno resultan ser ambiguos y se encuentran en constante debate. En este texto se emplea el término destino como referencia a los lugares a los que se dirigieron los sujetos de estudio en su proceso de emigración, el cual, en la mayoría de los casos, representó su lugar de residencia por largos periodos.

${ }^{3}$ Cuestionario Migrantes Devueltos por las Autoridades de Estados Unidos de la Encuesta de Migración de las Fronteras Norte y Sur (El Colegio de la Frontera Norte et al., 2020).
} 
después de la deportación desde Estados Unidos. El análisis privilegia la mirada en las categorías y los mecanismos de control del Estado sobre la movilidad humana, por ello se dialoga con autores que discuten sobre ciudadanía desde la perspectiva liberal y las aproximaciones biopolíticas (Collyer, 2012; De Génova, 2013; Fischer, 2013; Marshall, 1950) y también con literatura de corte empírico centrada en analizar los procesos de inclusión/exclusión en términos de la reinserción social en el país de origen después del retorno (voluntario o involuntario) y la relación con los recursos y capitales de los individuos (Cassarino, 2004; Contreras Velasco, 2016; Hualde et al., 2015; París et al., 2017; Pérez, 2014; Rivera, 2013).

El texto se ordena de la siguiente manera: en primer lugar, se presenta un breve resumen del andamiaje metodológico con enfoque cualitativo-inductivo; posteriormente, se presenta el marco analítico a través del cual se discuten los conceptos de movilidad, ciudadanía y deportación a la luz de los procesos de reinserción social en la frontera, con ello se introduce el análisis entre documentación y reinserción residencial y de vivienda, y la movilidad intraurbana. El artículo concluye que la condición de documentación influye en las posibilidades de inserción residencial y en el acceso a la vivienda, el no tener documentos de identidad se relaciona estrechamente con la ausencia de un domicilio fijo, lo que empujará a una condición de permanente movilidad o itinerancia intraurbana y ello a una mayor vigilancia y control por la policía.

\section{Metodología}

El diseño metodológico siguió las bases del método biográfico (Mallimaci \& Giménez, 2006). Se usó la noción de trayectoria de vida como un recurso heurístico que permitió introducir las dimensiones de tiempo, proceso y contexto en las historias de vida. Se definió la entrevista de corte biográfico como principal fuente de datos, acompañada de entrevistas informales y semi-estructuradas, así como de observación participante llevada a cabo en distintos espacios de la ciudad de Tijuana, Baja California, México, identificados previamente como los lugares de interacción y movilidad de la población de estudio.

Se logró un amplio recorrido en campo en diferentes etapas de la investigación distribuidas a lo largo de tres años entre 2015 y 2017. Se realizaron 68 entrevistas en total a la población objetivo de donde se extrajo una muestra de 23 casos. Esta muestra se definió así en función de dos criterios: el primero fue el acceso a las historias de vida y el seguimiento a sus procesos en la ciudad, lo que representó un reto metodológico al tratarse de población en condiciones de permanente movilidad intraurbana y en condición de calle (Tabla 1). A partir de esta condición, los casos se construyeron en torno a estas características: son individuos deportados desde Estados Unidos a México principalmente entre 2009 y 2017, residentes en la ciudad de Tijuana en un periodo no mayor a cinco años, aunque se incluyeron casos de más tiempo por la riqueza de la información. Se realizaron 17 entrevistas semiestructuradas a informantes clave como funcionarios de instituciones, directores o encargados de organizaciones sociales y/o activistas. 
Tabla 1. Trabajo y tipo de vivienda de las personas deportadas según documentación

\begin{tabular}{|c|c|c|c|c|c|c|c|}
\hline Documentos & Trabajo & Casa & Cuarto & Dpto. & Albergue & Calle & Total \\
\hline \multirow{4}{*}{ Con documentos } & Empleado Call Center & 4 & & 3 & & & 7 \\
\hline & Empresario & 1 & & & & & 1 \\
\hline & $\begin{array}{c}\text { Obrero en servicios de } \\
\text { construcción en Home } \\
\text { Depot }\end{array}$ & & & 1 & & & 1 \\
\hline & Maquila & 3 & & 1 & 1 & & 5 \\
\hline \multirow{3}{*}{ Sin documentos } & $\begin{array}{l}\text { Oficios de servicio } \\
\text { doméstico* }\end{array}$ & & 3 & & & & 3 \\
\hline & $\begin{array}{c}\text { Trabajadores por día/ } \\
\text { Central de abastos }\end{array}$ & & & & 4 & 1 & 5 \\
\hline & Talonero** & & & & & 1 & 1 \\
\hline Total & & 8 & 3 & 5 & 5 & 2 & 23 \\
\hline
\end{tabular}

*Tienen oficios como electricista, albañil, pintor, fontanero.

** Talonero es aquella persona que trabaja limpiando carros o pidiendo apoyo en las filas de autos que esperan el cruce fronterizo.

Fuente: elaboración propia.

\section{Reciudadanización, postdeportación y domicialización}

La vida de millones de inmigrantes mexicanos indocumentados en Estados Unidos muestra su otro rostro con la deportación de cientos de miles de ellos. Aun nacidos en México, las personas que son deportadas no recuperan los derechos de ciudadanía de manera automática con el retorno. Una vez en Tijuana, México, los deportados enfrentan obstáculos para obtener su documentación en distintos niveles del aparato burocrático del Estado, que hasta 2014, en una adición constitucional, considera el derecho a la identidad como una garantía ciudadana.

Schuster y Majidi (2013) así como Dingeman y Rumbaut (2010) han empleado el concepto postdeportación para enfatizar que el proceso que se vive luego de la deportación y el regreso al país de origen está marcado por el traslado de la carga simbólica ejercida sobre los migrantes indocumentados que no lograron obtener la ciudadanía en los lugares de destino, en este caso, en Estados Unidos, más todos los elementos que condujeron a su deportación, y también por aspectos subjetivos propios de la experiencia individual de cada sujeto.

El proceso de postdeportación, que busca establecer la conexión de los procesos y las experiencias vividas en cada contexto, estaría asociado a uno más amplio que podría ser denominado como reciudadanización, recuperación de la ciudadanía formal, es decir, de la relación de los individuos con el Estado de origen, que tiene como primer paso la recuperación de dichos documentos y un subsecuente proceso desigual de inserción o reinserción en las distintas dimensiones de la vida pública y privada, esto es: el trabajo, la vivienda, el acceso y movilidad en el espacio público, fundamentalmente. 
Como proceso, la reciudadanización se relaciona, por un lado, con el planteamiento de Fischer (2013) y Collyer (2012) acerca del distanciamiento con el Estado de origen y una ruptura de la relación individuo-Estado que ocurre en la emigración y la residencia en el país de "destino". Lo que Moreno (2014) denomina, a su vez, como "desciudadanización", en la que los individuos "desterritorializados", por su condición migratoria indocumentada en otro país, viven una especie de suspensión práctica de sus derechos en el país de origen, y son considerados "no ciudadanos" del Estado receptor.

Por otro lado, dicho proceso que ocurre en un nuevo lugar de residencia en el país de origen luego de la deportación está relacionado con aspectos que intervienen en la experiencia misma en el nuevo lugar de residencia, lo que se vincula tanto con aspectos estructurales propios del contexto de recepción, en el que entran en juego distintos actores y agentes gubernamentales y de la sociedad civil, quienes a nivel local actúan con discrecionalidad en los procesos de búsqueda de integración y apoyo local que llevan a cabo los individuos, creando desigualdades entre unos y otros. También la reciudadanización se vincula con aspectos de la experiencia individual, los capitales y recursos acumulados a lo largo de sus trayectorias vitales y de movilidad en los distintos contextos, es decir: en el proceso previo a la migración, en la migración y en la deportación (Pinillos, 2018).

En términos estructurales, los regímenes de ciudadanía, "herramienta analítica que condensa diversas aristas de un entramado en permanente tensión, no como un orden dado y fijo, por cuanto varían de acuerdo al tiempo y al espacio" (Argüello, 2019), cuentan con la documentación de identidad como un mecanismo de diferenciación del acceso y ejercicio de los derechos ciudadanos, no obstante las amplias discusiones en torno a la ciudadanía y la importancia de pensar este concepto van más allá de los marcos nacionales (Bosniak, 2001; Sassen, 2013). La visión clásica de ciudadanía de la perspectiva liberal, denominada también ciudadanía formal o política (Marshall et al., 1997), en la que se sostienen todas las líneas de análisis de la relación individuoEstado, y que a la luz de la experiencia de las poblaciones deportadas continúa siendo un mecanismo de diferenciación y control de la movilidad poblacional por el Estado, está fuertemente asociada a una noción de ciudadanía fija (Tilly, 1978), en virtud de la cual el establecimiento de un domicilio o residencia resulta imprescindible en la instrumentalización de los procedimientos que conduce la relación Estado-individuos. Se trata así de un proceso que podría ser denominado domicialización, en el cual la asignación de un domicilio posee un carácter dicotómico, esto es, no solo como derecho, sino que también se convierte en un requisito.

Esa dicotomía establece lo que se puede denominar como "la paradoja de la movilidad y la ciudadanía", particularmente desde la experiencia de las personas deportadas que se encuentran en Tijuana: la dificultad de obtener domicilio porque no se tiene documentos y, a su vez, la dificultad de obtener documentos porque no se tiene domicilio fijo. La movilidad es un término amplio y complejo que forma parte de una sólida discusión desde distintas disciplinas (Cresswell, 2006; Núñez \& Heyman, 2007; Zelinsky, 1971). En este documento se emplea el término movilidad para hacer referencia tanto a los procesos multicausales de la migración internacional y la migración interna (CIDH, 2015), como a las prácticas de desplazamiento locales y las dinámicas cotidianas de los habitantes de la ciudad que discute la geografía humana a través del concepto de movilidad espacial (Di Virgilio, 2011; Mendiola, 2012).

En Tijuana, paradójicamente, el no tener una residencia fija provoca que los individuos mantengan una itinerancia intraurbana, y con ello un mayor control y vigilancia de la policía (Pinillos, 2018). Este fenómeno, que definimos como paradójico, 
está relacionado a su vez con el planteamiento de Núñez y Heyman (2007) acerca de la relación dicotómica movilidad-atrapamiento. Su análisis basado en la experiencia de la frontera del lado estadounidense establece justamente que toda representación de movilidad implica una de "atrapamiento". Las formas de "libertad o flexibilidad" de movimiento, por ejemplo, en la franja fronteriza y al interior de las ciudades, conllevan formas de vigilancia que a su vez se traducen en formas de encerramiento.

Con lo anterior, se puede decir que el acceso y posesión de documentos se representa como el elemento que filtra y diferencia los procesos de inserción residencial y la movilidad en el espacio público, y se intersecta con los recursos de los propios deportados. ${ }^{4}$ Se trata de un proceso de diferenciación en la reinserción en México que parece tener como marco la visión de las personas deportadas como "indocumentadas" y como "legales liminales" (Menjívar, 2006) construida en Estados Unidos, pero con vías distintas según los recursos personales y las estrategias que se puedan activar o que se creen una vez en territorio mexicano.

El concepto de legalidad liminal, propuesto por Menjívar (2006), hace referencia a los espacios o áreas grises que surgen en los procesos de búsqueda de la ciudadanía formal de centroamericanos en Estados Unidos, que posibilitan las categorías legales establecidas en la misma ley y que los mantiene sometidos a un espectro limitado de posibilidades de inserción en las distintas dimensiones de la vida pública, impactando su vida en numerosos casos por tiempos prolongados. Esos procesos que ocurren en los lugares de emigración, como en el caso de estudio de Menjívar (2006), parecen persistir, aunque no con la amenaza de expulsión, en la experiencia postdeportación en México sin documentos de identidad mexicanos. La recuperación de los documentos que permita legitimar la ciudadanía formal implica seguir un camino que puede ser prolongado en el tiempo, entre otras razones por la discrecionalidad de los agentes del Estado en el nivel local. Lo que, así como lo plantea Menjívar (2006) para los contextos de emigración, tendrá un efecto en las vidas de las personas y en las distintas formas de reinserción y de pertenencia o no pertenencia en la nueva sociedad receptora, aunque sea este su propio país de origen (Pinillos, 2018).

La reinserción social es un concepto normativo, por cuanto es una categoría fundamentada en los marcos del pensamiento del Estado (Avallone, 2019) y se establece en función de lo que se espera sea logrado por las personas que ingresan a un territorio, París et al. (2019) la definen como el vínculo que las personas retornadas o deportadas intentan construir con las instituciones del país de origen. En los estudios sobre retorno y deportación se ha llegado a plantear que, por ejemplo, en el caso de quienes migraron en edades muy tempranas ni siquiera es posible hablar de una reinserción sino que, en todo caso, debiera ser una inserción en la consideración de que no existió una relación previa con el Estado ni con la sociedad del país de nacimiento. En ese sentido, la discusión de inserción/reinserción involucra tanto aspectos estructurales como subjetivos vinculados a la identidad, la pertenencia, las expectativas, entre otros. Aunque en las poblaciones deportadas el margen donde entran en juego las preferencias y las decisiones de los sujetos resulta ser pequeño, dada la falta de horizonte a mediano y largo alcance (Archer, 2000).

\footnotetext{
${ }^{4}$ Dichos recursos con que cuentan los individuos a los que se está haciendo mención son las redes sociales (Paris et al., 2017; Pérez, 2014) y capitales sociales o culturales, como la escolaridad y el idioma (Cassarino, 2004; Rivera, 2013) y la adquisición de conocimientos y habilidades en oficios y profesiones desempeñadas en Estados Unidos (Hualde et al., 2015; Pérez, 2014), principalmente.
} 
Para efectos de este análisis, se considera que la inserción social, así como lo plantea Rivera (2013) en sus estudios con población retornada, está mediada por la dinámica entre mercados de trabajo y modalidades de inserción social en las diferentes escalas, global y local, y en los niveles tanto estructural como individual, de manera que la experiencia de retorno es eminentemente un proceso diferenciado, debido a la estructura de oportunidades en la que un inmigrante o retornado se inserta y a la posibilidad de transformar tales estructuras o de diseñar mecanismos que posibiliten su inserción/reinserción (Rivera, 2013). De manera que el estudio de los procesos de inserción debe considerar tanto las condiciones y experiencias vividas en cada contexto de movilidad, como las características del contexto en sí mismo.

En Tijuana, la inserción o reinserción social está filtrada por los procesos de documentación. En el caso de las poblaciones deportadas, quienes logran tener los documentos de identidad de manera más rápida se insertan en trabajos menos precarios en el mercado de trabajo formal, en esos casos la participación de los agentes del mercado tiene una fuerte relación con esa relativa facilidad en el acceso a los documentos oficiales, y para aquellos que lo logran, en quienes se muestra una acumulación de desventajas en cuanto a la naturaleza de sus capitales sociales y culturales, y la condición de precarización vía las adicciones, esa inserción está ligada al acceso a los servicios de apoyo que brindan las organizaciones o asociaciones civiles. Es decir, el no tener documentos va a redundar en una mayor precarización en las condiciones de vida, lo que conllevará también dificultades para tener una vivienda digna y así mismo a procesos sociales de deterioro humano como agudización de los problemas de adicción y la indigencia (Pinillos, 2018).

\section{Inserción residencial y documentación}

El acceso a la vivienda es un elemento básico de la ciudadanía (Marshall, 1950) y una de las dimensiones de acceso a derechos sociales en el esquema inclusión-exclusión (Velázquez de la Parra, 1991). Los procesos de inserción residencial en población deportada en México representan una veta de exploración y análisis por ampliar. El estudio de Golash-Boza y Hondagneu-Sotelo (2013)con poblaciones de Guatemala, República Dominicana, Brasil y Jamaica plantea que una dificultad común en los procesos de reinserción social de centroamericanos deportados es el acceso a vivienda relacionado con el deterioro de las redes y los problemas económicos con los que regresan a sus países.

Esta dimensión de la ciudadanía coloca a los individuos deportados como sujetos del sistema de beneficencia de un Estado. La vivienda, como derecho, es una de las tareas por las que trabajan los gobiernos y constituye una de las prestaciones de los sindicatos modernos. En las ciudades fronterizas del norte de México se ha creado una infraestructura de albergue que las ha ido convirtiendo en ciudades receptoras de población en tránsito y más recientemente de población retornada.

En Tijuana existe una cada vez más reconocida oferta de organizaciones que brindan servicios y apoyo a la población migrante en diversas áreas, y que se han creado a través de su historia como ciudad receptora de migrantes en tránsito y más recientemente también de población retornada o deportada desde Estados Unidos. 
Uno de los servicios en los que se concentra la mayor oferta es el de alojamiento temporal.

La oferta de alojamiento se otorga a las personas a través de dos vías: una primera vía es el servicio de albergue que se ofrece como parte del Programa de Repatriación Humana (PRH $)^{5}$ del nivel federal que trabaja en conjunto con las oficinas de gobierno locales encargadas de atender a esta población que ha sido deportada desde Estados Unidos. Este servicio se ofrece en el ingreso al Módulo de Repatriación, allí algunas personas toman esta prestación y son canalizados a los albergues respectivos, quienes cuentan con redes familiares o sociales en Tijuana o algún recurso económico pagan por la renta de un cuarto o se dirigen a la vivienda de sus conocidos en la ciudad, en los relatos de los casos de estudio hay quienes afirman no haber recibido dicha información y otros que expresan haber tomado la opción no al momento de su llegada a Tijuana, sino en días posteriores a la llegada siempre que tengan consigo el certificado de deportación que se otorga en el módulo.

El certificado de deportación es el que permite el acceso a este servicio social. La población que puede ser beneficiaria se ordena de la siguiente manera: los hombres son canalizados a la Casa del Migrante, las mujeres a la Casa Madre Asunta, y los niños o menores a la Casa YMCA, organizaciones que forman parte de la denominada "Coalición de Atención al Migrante" y que trabajan en conjunto y sinergia con los programas de gobierno local. El tiempo de estancia es de únicamente 15 días. Por lo regular, estas organizaciones ofrecen asistencia humanitaria en varias áreas como alimentación, alojamiento, baño con agua caliente, ropa y calzado, servicio médico básico (primeros auxilios) y bolsa de trabajo. Este último es uno de los servicios que se ha ido incorporando para ampliar el margen de acción de estas organizaciones en el proceso de repatriación-reinserción en México.

Una segunda vía es la oferta de albergues que en los últimos años han dejado de ser lugares de paso para ser lugares de larga permanencia, y que se han construido principalmente en la zona centro y sectores aledaños. De esta opción se informan en sus primeros pasos en la ciudad. Se trata de lugares creados por organizaciones civiles de origen cristiano-evangélico y otras laicas, la mayoría de las cuales alberga a migrantes y deportados desde Estados Unidos. Pero ofrecen el servicio de alojamiento al público en general con un pago mínimo que los encargados llaman "cuota de recuperación", el cual oscila entre 10 y 30 pesos por noche. Estos se han creado como lugares de estancia corta, pero se han ido transformando en los lugares de residencia de esta población. La oferta de servicios varía entre unos y otros: alimentación, servicios de casilleros para guardar pertenencias básicas como documentos y algunos otros elementos, servicio de lavandería que se paga aparte de la cuota de recuperación. En la mayoría de ellos el acceso solo se permite a partir de las 16:00 horas, en casos muy reducidos se consiente

\footnotetext{
${ }^{5}$ El PRH es un programa dependiente del Instituto Nacional de Migración (INM) y para su funcionamiento ha instalado módulos de repatriación donde se da inicio por el Estado mexicano a la recepción de los connacionales. El PRH ofrece los siguientes servicios en 11 ciudades fronterizas: 1. Comunicación con el consulado; 2. Llamada telefónica con familiares; 3. Asistencia médica y psicológica; 4. Agua y alimentos; 5. Traslado a albergues o estaciones de transporte; 6 . Canalización a albergues temporales; y, 7 . Constancia de ingreso al país (INM, 2018). El objetivo general ha sido coordinar los servicios de atención inmediata para las personas repatriadas (París, 2010, p. 25). Los oficiales de migración registran en el sistema de repatriaciones a cada sujeto que llega después de haber sido deportado desde Estados Unidos, se otorga un certificado de deportación que permite identificarse en territorio mexicano y ser beneficiario de los apoyos que puedan existir para personas deportadas por las distintas instancias en la ciudad (albergues, comedores, etcétera) (Pinillos, 2018).
} 
permanecer a lo largo del día y, sobre todo, en casos extraordinarios, por ejemplo, en casos de personas que tienen alguna discapacidad física que impida su movimiento. En otros, con reglas y políticas de convivencia más flexibles y calidad del servicio menor, sí se permite la estancia durante las 24 horas. Sin embargo, por lo regular estos lugares permanecen vacíos durante el día porque los individuos salen a buscar trabajo y/o recursos para subsistir. Las dinámicas de los espacios de alojamiento pareciera que los empuja a ser itinerantes.

En la última década, los albergues tradicionales de la "Coalición de Atención al Migrante", pasaron de albergar principalmente a migrantes en tránsito a personas deportadas desde Estados Unidos (Coubès et al., 2015). En 2016, Velasco y colaboradores (2017) documentaron la existencia de 30 albergues $^{6}$ la mayoría de origen eclesiástico que atendieron a 2677 personas por día. Para el caso de buena parte de la población deportada, estos albergues constituyen un espacio de llegada para luego dirigirse a otro lugar, ya sea el de origen, o bien instalarse en la ciudad. Para acceder a una vivienda en renta o comprada en Tijuana se requiere presentar documento de identidad, además de tener redes de confianza y un ingreso más o menos estable (Pinillos, 2018).

\section{Modalidades de vivienda luego de la deportación en Tijuana y documentos de identidad}

A partir de los casos de estudio en los que se basa este artículo se pueden identificar dos modalidades o grupos residenciales. Un primer grupo de 13 individuos que viven en casas o departamentos rentados por ellos mismos, aunque la mayoría apoyados económicamente por sus familiares en Estados Unidos y en menor medida por los del lado mexicano, lo que les permite mantener activas las redes transfronterizas; todos estos, de acuerdo con sus relatos, cuentan con documentos y con trabajos más formales, como call centers, maquilas y microempresas propias como locales de tatuajes y comercios de comida rápida. Algunos comparten su residencia con algún miembro de la familia o viven solos. ${ }^{7}$

Un segundo grupo de 10 personas, de las cuales tres viven en pequeños cuartos rentados, cinco en albergues y dos en la calle, se ocupan en trabajos informales y precarios en mercados y centrales de abasto, además la mayoría de ellos no cuentan con documentos. En este último caso, pareciera no existir un margen concreto entre las formas de residencia. En plazos muy cortos y, de acuerdo con los recursos con que se cuente, en una semana las personas pueden residir en un cuarto, luego en un albergue y algunos días en la calle. La vida en la calle está fuertemente relacionada con el consumo de drogas y alcohol y una profunda estigmatización (Albicker \& Velasco, 2016) que los vuelve sujetos de persecución policial (Contreras Velasco, 2016) y contribuye al ausentismo en el empleo.

\footnotetext{
${ }^{6}$ Este número refleja un incremento inusitado en el año 2016 debido a la inmigración de un flujo importante de población haitiana y desplazados mexicanos en búsqueda de asilo en Estados Unidos.

${ }^{7}$ Es importante mencionar que por efectos metodológicos y de acceso, por tratarse de espacios privados, no se realizó una observación directa de este tipo de vivienda.
} 
En el acceso a un lugar para pernoctar en Tijuana entran en juego aspectos objetivos y subjetivos. En lo objetivo están los tiempos y plazos que normativamente se establecen como marcos para el otorgamiento de servicios y apoyos de atención ante el fenómeno, 15 días de alojamiento en las organizaciones tradicionales. Además, la ley de migración considera como migrante a aquella persona que no lleva más de 6 meses de estancia en la ciudad. En el plano subjetivo se encuentran las percepciones y preferencias de los sujetos. En los casos de estudio se tiene, por ejemplo, la consideración del albergue como lugar seguro y limpio. El relato de Sergio ilustra este aspecto:

(...) prefiero el albergue del Toro porque está limpio, nada más que lo malo, dan palabra [servicio religioso], son como tres horas, cuatro horas, y a las 10 de la noche llegan los que dan la palabra y este (...) imagínate, viene acabando como a la 1 de la mañana y vienen dando de cenar, en veces varían, a las 7 de la noche, y con El Grande [albergue] no, porque en El Grande dormimos en el suelo, con una sábana nada más y el puro frío, y el frío (...) (Sergio, 2017).

Y de la calle como lugar que permite los vicios, el desenfreno, lo que puede representar un riesgo. Pero también ocurre de manera contraria, el albergue es, para algunos, el lugar de represión, de discriminación, de coartación de su libertad, y en algunos casos un lugar sucio: "allá es pura mentira que dan comida, eso dan lo que quieren y no a todos, todo hay que pagar, yo cuando quiero tomar no me quedo ahí porque está prohibido" (Ernesto, 2017).

De esta manera, el albergue es un recurso básico común en la trayectoria de retorno y los intentos de la inserción residencial de los individuos deportados en Tijuana. Si se piensa en el proceso, un punto inicial es el servicio de albergue, que como un derecho social se establece en el PRH como medida asistencial y temporal, por medio de las organizaciones locales de la sociedad civil.

Posterior a los primeros pasos para acceder a un lugar para pernoctar, se expanden y crean otras formas de residencia que estarán asociadas a las demás dimensiones básicas (trabajo, ocio, expectativas). La posibilidad de acceso a condiciones dignas de vivienda dependerá tanto de la posibilidad de recuperar su documentación de identidad y del tiempo en que esto pueda ocurrir, así como de la obtención de recursos suficientes y las preferencias de los individuos de permanecer o no en la ciudad y de qué manera. En estas múltiples formas de instalación residencial surge de nuevo el albergue, ahora como la constitución de un tipo de vivienda para la población deportada, es decir, el albergue pasa de concebirse solo como un lugar "temporal", para constituirse como el lugar en el que se vive, en este plano entran en juego los albergues de organizaciones religiosas y civiles o privados que abren sus puertas a todo tipo de población sin domicilio fijo.

\section{Los albergues como el lugar donde se habita y la calle como expresión de resistencia}

Los albergues en Tijuana se han convertido en el lugar de residencia de un sector específico de la población deportada, en el que realizan una buena parte de sus actividades diarias y de reproducción social: alimentación, limpieza, ocio, establecimiento de relaciones interpersonales, sobre todo por la prolongación de los 
tiempos de permanencia, entre los casos entrevistados y de acuerdo con lo narrado por los encargados de 10 albergues ubicados en la zona norte de la ciudad, hay personas que a mayo de 2017 tenían periodos de permanencia que superaban los cinco años (Pinillos, 2018).

La permanencia prolongada en los albergues es resultado de un conjunto de factores o aspectos entre los cuales se pueden citar la ausencia o limitación de recursos económicos y materiales, de redes familiares sólidas, así como de elementos subjetivos como el deseo de libertad o de seguridad. Es decir, las preferencias y expectativas de los individuos estarán siempre influenciadas por su condición de expulsados desde Estados Unidos: haber vivido en dicho país y el estigma sobre la imagen del deportado, por ejemplo, afectarán las posibilidades de acceder a recursos y servicios locales. Todo ello se intersectará con el hecho de tener o no tener documentos. Aunque no todos aquellos que son usuarios de los albergues se encuentran sin documentos de identidad mexicanos, lo cierto es que el que no sea un requisito tener un documento de identidad para tomar el servicio contribuye a que estos lugares sean una de las principales opciones para la población deportada indocumentada y sin recursos para rentar una vivienda.

La movilidad entre albergue y calle queda establecida en los relatos de los sujetos. Olivo y Gabriel, deportados desde Estados Unidos, son hombres que combinaban el lugar de dormir entre los albergues y la calle en un espacio cerca al Hospital General de Tijuana, dicen que prefieren dormir en la calle a falta de recursos para pagar un cuarto, y dado que no admiten el mal trato que dicen recibir en los albergues:

(...) la comida la cobran toda, en realidad se paga más de lo que dicen, y es mala la comida, pocas veces dan buenas cosas, los servidores son groseros. En el Ejército de Salvación tienen guardadas buenas cosas para ofrecer de comida, pero no las quieren dar La Roca del Alfarero es bueno (Olivo y Gabriel, 2017).

Álvaro, deportado en 2014, soltero, quien duerme en el albergue Casa del Emigrante el Aposento, ${ }^{8}$ comenta:

(...) aquí vivimos muchos de quienes estuvimos en El Bordo, ${ }^{9}$ yo prefiero dormir en este lugar porque es más flexible, los horarios de entrada y de salida, porque no obligan a nadie a escuchar la palabra de Dios, y porque en los otros lugares parece que fueran cárceles, y los servidores te tratan muy mal (...) (Álvaro, 2016).

Hay así una consciencia y queja sobre el trato que reciben y sobre las condiciones en las que se encuentran. Elementos subjetivos como la dignidad son los recursos que quedan en medio de los límites de la precariedad. Además, la continua comparación con el imaginario de la vida en "el otro lado" conduce a una suerte de inconformismo

\footnotetext{
${ }^{8}$ Un lugar que está en la Zona Norte de Tijuana, muy cerca de la zona para la prostitución de la ciudad y que permanece cerrado todo el tiempo con candado, un lugar oscuro con un olor profundo a cigarrillo, en el que se encuentran a la entrada unas máquinas de juegos y a lo largo de un pasillo unos cuartos con unas colchonetas, que parecen más unos tapetes, donde duermen las personas.

9 "El Bordo" constituía el espacio "a lo largo de la canalización del Río Tijuana en que residían entre 700 y 1000 personas, distribuidas en 118 ñongos, 25 hoyos, 7 alcantarillas y 10 puentes; también hay quienes se asientan a la intemperie a lo largo de los 2 kilómetros de laderas, túneles y pendientes" (Velasco \& Coubés, 2013).
} 
permanente sobre lo que se tiene o pueda tener en el presente. La reducción del consumo que, según los sujetos, podía tenerse en un país como Estados Unidos, se vuelve también un elemento permanente de comparación que influye en las decisiones que se toman en el presente.

La posibilidad de elegir sobre oferta de albergues o para tener un lugar donde dormir y las condiciones deseadas dependerán en buena parte de las posibilidades de trabajo que encuentren en el día, pero también de sus deseos, del establecimiento de sus prioridades. Los albergues y comedores, en todo caso, funcionan como dispositivos o como mecanismos de subvención para la reproducción social de esta población deportada, indigente y móvil en la frontera. Una población que se compone principalmente por población masculina.

En lo cotidiano, las organizaciones que brindan apoyo a esta población deportada e itinerante establecen, a su vez, desigualdades y diferenciaciones entre los beneficiarios o usuarios. Por ejemplo, en uno de los albergues con mayor capacidad de atención (180 personas por noche en la sección donde se alojan los hombres, también hay una sección para 12 mujeres) se ofrece una cama (litera) para quienes tienen mayor tiempo de estancia en el lugar y se da preferencia a quienes paguen por semanas o por periodos de varios días. Mientras que aquellos que llegan por primera vez es probable que tengan que dormir en colchonetas ubicadas en el piso:

(...) las camas se las damos a aquellos que ya se han ganado la confianza de las personas encargadas del albergue y que son constantes allí. Son 60 literas, es decir, 120 camas, la capacidad del albergue es de 180 (...) (Moisés, 2016).

Así, los albergues parecen funcionar en la formación de estilos de vida que se escapan del ideal tradicional de la vida normalizada y estandarizada, de la vida "exitosa", de la inserción plena, de la noción de hogar y de familia, de la construcción de la vida cotidiana y pone en consideración el tema de los cuidados, que merece ser analizado más a fondo, por cuanto expresa la profunda complejidad de los procesos sociales que se desarrollan en la reconstrucción de la vida en un contexto fronterizo particular como es el de Tijuana después de la deportación. Hace falta el establecimiento de una visión que cuestione el paradigma de inclusión-inserción social de la población en condición de deportación en los tiempos de complejas movilidades en múltiples direcciones.

Todas estas condiciones conllevarán al no establecimiento de los márgenes entre la vida en el albergue y en la calle, pues la calle también representa un espacio ocupado para dormir por una parte de la población luego de ser deportada a Tijuana que se prolonga en el tiempo. Tal realidad parece estar asociada a procesos de deterioro de las condiciones físicas y mentales que se desencadenan tras periodos prolongados de itinerancia, así como de rupturas con los vínculos y los lazos personales y familiares.

Dichos procesos de deterioro, asociados a la itinerancia en la ciudad, fueron observados en distintos individuos durante los recorridos en campo a lo largo de un año, particularmente en tres de los entrevistados:

- Ernesto, deportado en 2016, quien vivía en un albergue desde entonces, trabajaba pelando tomatillo y dedicaba sus horas de ocio a jugar ajedrez en el parque Vicente Guerrero en la zona Centro de Tijuana y a consumir sustancias. Hacia junio de 2017 se encontraba viviendo en la calle en condiciones en las que parecía no tener salud mental; 
- Porfirio, deportado en 2013, quien trabajaba en el mercado de la zona Centro en el pelado de tomatillo y regularmente dormía en el albergue La Roca, pero en diversas ocasiones decidió quedarse en la calle para ingerir bebidas alcohólicas y en alguna de esas ocasiones llegó a ser "picado" (apuñalado) al quedarse a dormir en la calle; $y$

- Calixto, quien, de todos los individuos entrevistados, es el que en peores condiciones físicas se encontraba la última vez que se logró tener contacto con él. Con 36 años al momento de la entrevista, es el caso de deterioro más cercano al que se pudo dar mayor seguimiento durante la inmersión en campo. Deportado desde Estados Unidos por Tijuana en 2008 a causa de problemas relacionados con consumo de sustancias, era un usuario del Albergue La Roca. Durante el periodo en el que se pudo establecer contacto con él (abril a diciembre en 2016) se dio seguimiento a su movilidad entre varios albergues y lugares para dormir, así como al proceso de deterioro de sus condiciones de vida evidentes: algunas veces dormía en el albergue La Roca del Alfarero, uno de los albergues más grandes de la ciudad. Calixto llegó a La Roca después de haber estado en otros albergues como el Ejército de Salvación en el momento posterior a su deportación, en donde estuvo encargado de la cocina, pero luego de unas semanas decidió buscar otro lugar para dormir. Hacia mayo de 2016 trabajaba desde las 9:00 de la mañana hasta las 14:00 horas en un micro call center de una organización civil creada por deportados, donde brindan capacitación a personas que hablan inglés en el servicio de atención al cliente con llamadas, por un pago de 100 pesos al día. En la tarde era ayudante en la cocina de La Roca, a partir de las 16:00 horas. Algunas otras veces Calixto no se quedaba en el albergue cuando decidía tomar licor y consumir sustancias. Para octubre de 2016, Calixto había renunciado al call center y desde ese tiempo no regresó al albergue, comenzó a vivir en la calle y la última vez que se pudo tener contacto con él estaba debilitado físicamente, y su capacidad para sostener una conversación estaba también limitada.

De acuerdo con lo anterior, la inserción residencial, un aspecto de la reproducción y la construcción de la familia como institución ordenadora social del tipo que sea (casa propia o rentada, o albergue), en el caso de la población deportada en Tijuana va a redundar en formas de vida diferenciadas y desiguales, formas de sociabilidad y de reproducción social particulares.

Esas complejas movilidades en una ciudad fronteriza: atrapamientos en la movilidad internacional y creación de circuitos de alta movilidad al interior de las ciudades, se pueden comprender a la luz de la no-documentación y la inestabilidad residencial, puesto que la conjunción de estos dos elementos lleva a una mayor itinerancia urbana que provoca, a su vez, un aumento de la vigilancia de los agentes de seguridad del gobierno, en particular de la Policía Municipal. Aunque la vigilancia de la policía se ejerce en Tijuana sobre distintos tipos de población, esto es, no solo sobre la población en situación de calle, la que se despliega sobre las personas que están en constante movilidad en las calles y sin documentos de identidad, se legitima y ocurre de manera más dura y frecuente, como lo han mostrado autores como Sayad (2011), Das y Poole (2004) y Contreras Velasco (2016). 


\section{Itinerancia intraurbana y documentación}

La relación entre itinerancia y documentación de identidad tiene varias aristas analíticas. Por un lado, esa itinerancia puede constituir parte de la flexibilidad que les exige el tipo de trabajos precarios a los que accede un sector de los deportados (Pinillos, 2018). A la vez, esa misma condición de itinerantes sin documentos constituye parte de su vulnerabilidad como residentes móviles de esta ciudad fronteriza, que los somete a mayor vigilancia de los agentes de la policía.

La condición de itinerancia e indocumentación se retroalimenta mutuamente, porque estar en movimiento constante los expone a la posibilidad de pérdida de los documentos que logran obtener, mientras que no tener documentos los obliga a estar en movimiento constante, huyendo de la policía o tratando de pasar inadvertidos en clandestinidad, lo que los coloca en situación de alta precariedad. Los casos de estudio reiteran constantemente la experiencia de detención arbitraria por la policía municipal. Las razones dadas son por no tener documentos y por su apariencia. Ello coincide con otros estudios como el de Velasco y Coubès (2013, p. 22, 25), en el que se encontró que los habitantes de "El Bordo", 91\% expresó haber regresado de Estados Unidos (91.5\% por deportación), 93.5\% declaró que había sido detenido por la policía en la ciudad de Tijuana en los últimos 15 días, de los cuales 19\% reportó haber sido detenido tres veces o más, es decir más de una vez por semana.

Estos hallazgos tienen que ser analizados a la luz de la tesis de Torpey (1998) sobre movilidad libre como una condición de la ciudadanía. En la constitución mexicana está consagrado como un derecho fundamental el libre tránsito, sin embargo, los reglamentos locales condicionan ese libre tránsito a la domicialización. Por ejemplo, el reglamento llamado Bando de Policía y Gobierno del municipio de Tijuana considera como habitante solo a aquellos que tengan un domicilio, ya sea temporal o permanente, y establece como infracción dormir en el espacio público. ${ }^{10}$

La ciudad de Tijuana tiene un flujo intenso de población en sentidos múltiples (50 000 cruces internacionales regulares por día). En esos cruces los flujos de personas devueltas, como denomina la Encuesta sobre Migración en la Frontera Norte (EMIF) ${ }^{11}$ a la población que retorna de manera involuntaria desde Estados Unidos a México, han cobrado cada vez mayor visibilidad. ${ }^{12}$ De alguna forma, la frontera mexicana es el prototipo del escenario que dibuja Torpey (1998) al hablar de la relevancia exagerada que han tomado los pasaportes y documentos de identidad en el modelo de Estado, produciendo burocracias y lógicas de intenso control y vigilancia.

El control sobre la movilidad interna se ha agudizado con la crisis de violencia asociada al crimen organizado, lo que legitima las estrategias del Estado para enfrentarlo. ${ }^{13}$ Las detenciones y la relación violenta que ejerce la fuerza pública sobre los cuerpos de los sujetos en los espacios públicos, particularmente de aquellos que se encuentran en situación

\footnotetext{
${ }^{10}$ Ver artículos 13 y 72 del Bando de Policía y Gobierno del municipio de Tijuana, 2017 (XXII Ayuntamiento Tijuana, 2017).

${ }^{11}$ Encuesta de Migración de la Frontera Norte y Sur.

12 Una idea del volumen lo da la siguiente información. En los años de 2013 y 2012 se realizaron 4.6 millones de devoluciones por la frontera norte de México (Velasco \& Coubès, 2013).

${ }^{13}$ En los dos últimos sexenios presidenciales la cantidad de muertos por violencia suma 234000 (Hernández Borbolla, 2017).
} 
de calle, forma parte, como lo mencionan Bloch y Schuster (2005), de una representación simbólica que legitima el miedo, el gasto elevado en términos de recursos públicos que implica desplegar unidades policiales y agentes en distintos puntos de la ciudad.

Pero ese control tiene consecuencias serias sobre la seguridad de la población y mucho más para la población deportada en esta frontera. No tener documentos de identidad, aun siendo mexicanos, los convierte en sujetos de sospecha sobre los que se ejerce constante vigilancia, como sucedía en su estancia como migrantes indocumentados en Estados Unidos. El relato de Sergio destaca a la policía como un elemento importante en las dinámicas y los itinerarios de vida de la población que llega en busca de trabajo a esta ciudad, porque funge como un agente de control y exclusión, que limita la movilidad de los sujetos al interior de la misma ciudad, y garantiza el circuito de precariedad laboral y social a la que se enfrentan.

Siempre adelanto la renta un mes, porque en veces uno no sabe, a veces el trabajo no hay o cualquier cosa, trato de vivir bien, no tan mugroso porque aquí la policía te ven mugroso, te levanta (...) Y luego la policía allá nos golpea adentro de las celdas y yo como soy gay hay un policía que siempre me trae y siempre que me agarra, me da 36 horas, yo no veo al juez y ya el policía adentro me comienza a golpear, yo no (...) ni para poner un reporte con derechos humanos porque me van a traer al policía. El juez nos califica en la forma como te ve vestido (Sergio, 2017).

El caso de Sergio muestra cómo los mecanismos de control de la movilidad intraurbana están cruzados por los procesos de estigmatización. Los agentes estatales de la seguridad pública comparten los prejuicios sobre los deportados a partir de su apariencia y la idea del fracaso que se construye a través de los medios de comunicación y los programas que dirigen las instituciones gubernamentales en donde se crean discursos y acciones que discriminan a los deportados. Esta vigilancia se acompaña de un abuso y extracción de los pocos recursos económicos que alcanzan a tener. Los relatos de quienes viven en albergues, en cuartos y en la calle coinciden en la denuncia del robo de su dinero y pertenencias en cada detención policiaca. Por lo que entran en una especie de espiral de precarización, del cual es muy difícil salir.

En definitiva, la tenencia de documentación de identidad, no solo los pasaportes, es una fuente de desigualdad entre la población deportada, que se entrecruza con otras ausencias de capitales como la escolaridad y las habilidades lingüísticas que limitan su movilidad interna e internacional. Aun los deportados que han logrado documentarse debidamente enfrentan esa vigilancia, pero con otros capitales sociales y culturales. En estos casos, no solo son los documentos sino también su capital humano y el conocimiento de sus derechos lo que permite que el abuso de la autoridad pueda enfrentarse de manera distinta.

En las prácticas de la policía también se observa la constitución del Estado mismo, como lo señala Contreras Velasco (2016) al documentar la violencia que la policía municipal ejerce sobre la población que vive en la calle de la ciudad de Tijuana. Es decir, la fuerza pública es la institución a través de la cual el Estado tiene dominio y relación en los espacios públicos, sin embargo, en la calle, queda limitada su capacidad de acción (Contreras Velasco, 2016). Esta condición de permanente vigilancia sobre los individuos deportados agudiza su precariedad, profundizando la marginalidad que se ha ido consolidando en un proceso conexo de "movilidad-encerramiento" (Núñez \& Heyman, 2007) tanto en México como en Estados Unidos. 


\section{Conclusiones}

Los hallazgos de la investigación en la que se sustenta este texto muestran que un gran obstáculo de la reinserción social de los deportados es la dificultad en el acceso a los documentos de identidad y constituye una importante fuente de desigualdades entre los deportados que se enlaza con sus opciones de empleo y vivienda. La idea de obstáculo emerge tanto de la noción normativa de la reinserción que define también la existencia de elementos que facilitan o dificultan los procesos que los individuos deportados "deben" llevar a cabo en Tijuana; como de los discursos y los relatos de los sujetos entrevistados y de los miembros de las organizaciones de la sociedad civil involucradas en el contexto de recepción de población migrante y deportada.

Los estudios sobre retorno y deportación dejan en claro la importancia de los elementos contextuales para entender este proceso. En ese sentido, un aspecto importante a considerar es la asimetría entre estos países, como lo describe Rivera (2013), ello evidentemente reduce las posibilidades de movilidad, de readaptación y reajuste de expectativas, que se definen en relación con los recursos encontrados en ambos países (Rivera, 2013, p. 60), lo que se observa con mayor contundencia en las regiones fronterizas donde estos flujos e intercambios son cotidianos. Por lo tanto, es preciso ubicar los procesos de reinserción de la población deportada desde Estados Unidos en Tijuana como ciudad fronteriza, con el propósito de comprender las razones y las expectativas de los sujetos para establecer su permanencia en la ciudad.

En términos de inserción residencial, se tienen distintas formas de vivienda para esta población relacionadas con el acceso a la documentación en Tijuana. Como ya se dijo, la visión domiciliada del ciudadano responde a la de una ciudadanía fija espacialmente. Tener residencia requiere tener documentos y viceversa. Generalmente, las personas que acceden a la documentación tienen mayor facilidad para acceder a una vivienda en renta. La observación de las condiciones de vivienda y residencia de la población de estudio representa una veta abierta de análisis.

La dificultad de acceso a vivienda de una parte de la población deportada, fundamentalmente de aquellos que viven en albergues o en la calle, es un problema recurrente. La condición de deportación en los tiempos de complejas movilidades en múltiples direcciones cuestiona la idea de hogar. Las personas que viven en cuartos, albergues o la calle, tienen una forma de vivir en la que siempre se está sin lugar fijo de residencia.

Las personas sin documentación dependen más de la oferta de los albergues, por las normas de funcionamiento de estos lugares ello conduce a una circulación constante. En sus itinerarios residenciales se incluye la calle o áreas públicas, hoteles o cuartos de renta por periodos cortos. La residencia en los albergues pasa de ser temporal a ser permanente, como estilos de vida o formas distintas al estilo sedentario o domiciliado y configura cambios en las visiones estandarizadas de la vida. Esto es un fenómeno muy importante de la ciudad de Tijuana, en donde la llegada de distintas poblaciones migrantes y/o con experiencia de deportación, principalmente desde 2008, ha conducido a la creación de lo que se podría llamar una infraestructura social de atención con la participación de múltiples actores sociales. Considerar estas configuraciones residenciales novedosas de las ciudades fronterizas con alta movilidad conduce a cuestionar las visiones homogéneas y generalizadas de inclusión-inserción social esperada sobre una población con condición de desplazamiento forzado por deportación. 
La no residencia fija está asociada a una continua movilidad entre albergues que se extiende a una movilidad en los espacios públicos. No tener documentos y no tener residencia puede conllevar a una itinerancia diaria en los sujetos, lo que los ubica como sujetos de sospecha sobre quienes se ejerce una vigilancia permanente por la fuerza pública, similar a lo que ocurría en su residencia en Estados Unidos.

Parece que los trabajos más precarios requirieran esta fuerza laboral móvil que se ajusta a las formas de contratación flexible y completamente informal. Pero esa itinerancia se penaliza de diferentes formas en los encuentros con la fuerza pública, con el encierro o con un pago. Las detenciones dificultan el acceso al trabajo y precarizan más esta fuerza de trabajo y la mantienen sometida y explotada.

Hay varias consecuencias de esta condición humana que pueden ser interpretadas de distintas formas. Una podría ser la deshumanización y pérdida de ciudadanía de las personas migrantes quienes en deportación permanecen con la ausencia de una vivienda, una familia separada, un trabajo y en condiciones sumamente precarias. A la vez, se podría leer como una condición de vida en la "antidisciplina", en la medida que las formas de control: documentación, vivienda y trabajo no son vías de registro en una parte de la población. No obstante, la agencia que traduciría la "antidisciplina" es relativa, porque estos hallazgos indican que aun en la precarización alta, estas personas están ligadas a formas de explotación, en los mercados, en los oficios informales, usados como clientela política o bien como sujetos de la compasión de los sectores que atienden a la población migrante en la ciudad. La precariedad de esta población deportada es funcional económica y socialmente.

Se puede dilucidar la existencia de una libertad condicionada y regulada por el Estado, que pareciera tener como único lugar de encuentro con esta población el que logra a través de la policía. La inmovilidad o atrapamiento — discutidos por Núñez y Heyman (2007) - que pueden experimentar los deportados por diferentes lapsos en sus vidas, se vuelve un signo más de pérdida de ciudadanía. Como lo plantean Das y Poole (2004) al referirse a los documentos de identidad como la forma en que el Estado se muestra en su dualidad lejos/cerca, una forma de lenguaje y mecanismo para comunicar su capacidad de controlar y regular a la sociedad y condicionar la ciudadanía. La vigilancia sobre la movilidad interna de la población se vuelve en una amenaza para la libre movilidad de toda la población. Una paradoja de aparente libertad de movimiento, pero en la que queda develada todo el poder del Estado y el control y la desigualdad que dicho poder construye a través del sostenimiento de la noción de ciudadanía formal y fija.

\section{Referencias}

Albicker, S. L. \& Velasco, L. (2016). Deportación y estigma en la frontera México-Estados Unidos: Atrapados en Tijuana. Norteamérica, revista académica del CISAN-UNAM, 11(1), 99-119. https://doi.org/10.20999/nam.2016.a004

Archer, M. S. (2000). Being human: The problem of agency. Cambridge University Press.

Argüello, S. (2019). De la politización a los regímenes de ciudadanía. Ajustes analíticos para estudiar las disputas por los derechos sexuales. Estudios Sociológicos de El Colegio de México, 37(110), 489-503. https://doi.org/10.24201/es.2019v37n110.1790 
Avallone, G. (2019). La movilidad humana en la red de las palabras de Estado. $R E-$ MHU: Revista Interdisciplinar da Mobilidade Humana, 27(57), 25-42. https://doi. org/10.1590/1980-85852503880005703

Bloch, A. \& Schuster, L. (2005). At the extremes of exclusion: deportation, detention and dispersal. Ethnic and Racial Studies, 28(3), 491-512. https://doi. org/10.1080/0141987042000337858

Bosniak, L. (2001). Denationalizing citizenship. En D. Klusmeyer \& T. A. Aleinikoff (Eds.), Citizenship today: global perspectives and practices carnegie endowment for international peace (pp. 237-252). Carnegie Endowment for International Peace.

Cassarino, J.-P. (2004). Theorising return migration: The conceptual approach to return migrants revisited. International Journal on Multicultural Societies (IJMS), 6(2), 253-279. https://papers.ssrn.com/abstract $=1730637$

Comisión Interamericana de Derechos Humanos (CIDH). (2015). Derechos humanos de migrantes, refugiados, apátridas, víctimas de trata de personas y desplazados internos: Normas y Estándares del Sistema Interamericano de Derechos Humanos (Movilidad humana éstandares interamericanos). Comisión Internamericana de Derechos Humanos. OEA/Ser.L/V/II.Doc. 46/15

Collyer, M. (2012). Deportation and the micropolitics of exclusion: The rise of removals from the uk to Sri Lanka. Geopolitics, 17(2), 276-292. https://doi.org/10.10 $80 / 14650045.2011 .562940$

Contreras Velasco, O. (2016). Vivir en los márgenes del Estado: Un estudio en la frontera México-Estados Unidos. Región y Sociedad, 28(65), 235-262.

Coubès, M.-L., Gonzalez Fagoaga, E., Mejía Arango, S., Nevarez Sanchez, R. \& Velasco Ortiz, L. (2015). Estudio sobre los usuarios del Desayunador Salesiano del Padre Chava. El Colegio de la Frontera Norte/Proyecto Salesiano Tijuana, A. C. https://www. colef.mx/estudiosdeelcolef/estudio-sobre-los-usuarios-del-desayunador-salesiano-del-padre-chava/

Cresswell, T. (2006). On the move: mobility in the modern Western world. Routledge.

Das, V. \& Poole, D. (Eds.). (2004). Anthropology in the margins of the state. School of American Research Press.

De Génova, N. (2002). Migrant "Illegality" and Deportability in Everyday Life. Annual Review of Anthropology, 31, 419-447.

De Génova, N. (2013). Spectacles of migrant 'illegality': The scene of exclusion, the obscene of inclusion. Ethnic and Racial Studies, 36(7), 1180-1198. https://doi.or $\mathrm{g} / 10.1080 / 01419870.2013 .783710$

Di Virgilio, M. M. (2011). La movilidad residencial: Una preocupación sociológica. Territorios, 2(25), 173-190.

Dingeman, M. K. \& Rumbaut, R. G. (2010). The immigration-crime nexus and post-deportation experiences: En/counterings stereotypes in Southern California and El Salvador. University of La Verne Law Review, 31(2), 363-402.

El Colegio de la Frontera Norte, Unidad de Política Migratoria, Registro e Identidad de Personas, Consejo Nacional de Población, Consejo Nacional para Prevenir la Discriminación, Secretaría del Trabajo y Previsión Social, Secretaría de Relaciones Exteriores, Secretaría de Bienestar. (2020). Encuesta sobre Migración en la Frontera Norte de México. www.colef.mx/emi 
Fischer, N. (2013). Bodies at the border: The medical protection of immigrants in a French immigration detention centre. Ethnic and Racial Studies, 36(7), 1162-1179. https://doi.org/10.1080/01419870.2013.783708

Golash-Boza, T., \& Hondagneu-Sotelo, P. (2013). Latino immigrant men and the deportation crisis: a gendered racial removal program. Latino Studies, 11, 271-292. https://doi.org/10.1057/lst.2013.14

Hernández Borbolla, M. (2017, noviembre 23). Peña y Calderón suman 234 mil muertos y 2017 es oficialmente el año más violento en la historia reciente de México. The Huffinfton Post México. http://www.huffingtonpost.com.mx/2017/11/23/ pena-y-calderon-suman-234-mil-muertos-y-2017-es-oficialmente-el-año-mas-violento-en-la-historia-reciente-de-mexico_a_23285694/

Hualde, A., Jurado, M., \& Tolentino, H. (2015). El significado del trabajo y su relación con la precariedad en los call centers. En R. Guadarrama, A. Hualde \& S. López (Coords.), La precariedad laboral en México. Dimensiones, dinámicas y significados (pp. 331-356). El Colegio de la Frontera Norte/unM-Cuajimalpa.

Instituto Nacional de Migración (INM). (2018). Programa de Repatriación. http://www. gob.mx/inm/acciones-y-programas/programa-de-repatriacion-12469

Mallimaci, F. \& Giménez Béliveau, V. (2006). Historia de vida y métodos biográficos. En I. Vasilachis de Gialdino (Ed.), Estrategias de investigación cualitativa. Gedisa

Marshall, T. H. (1950). Citizenship and social class: and other essays. Cambridge University Press.

Marshall, T. H., Casado, M. T. \& Noya Miranda, F. J. (1997). Ciudadanía y clase social. Reis: Revista Española de Investigaciones Sociológicas, 79, 297-344. https://doi. org/10.2307/40184017

Mendiola, I. (2012). Regímenes de movilidad y domesticación del espacio. Política y Sociedad, 49(3), 433-452. https://doi.org/10.5209/rev_POSO.2012.v49.n3.38549

Menjívar, C. (2006). Liminal legality: Salvadoran and Guatemalan immigrants' lives in the United States. American Journal of Sociology, 111(4), 999-1037. https://doi. org/10.1086/499509

Moreno, H. C. (2014). Desciudadanización y estado de excepción. Andamios, Revista de Investigación Social, 11(24), 125-148. https://doi.org/10.29092/uacm.v11i24.235

Núñez, G. G., \& Heyman, J. McC. (2007). Entrapment processes and immigrant communities in a time of heightened border vigilance. Human Organization, 66(4), 354-365. https://www.jstor.org/stable/44127414?seq=1

París, M. D. (2010). Procesos de repatriación. Experiencias de las personas devueltas a México por las autoridades estadounidenses (documento de trabajo). Woodrow Wilson International Center for Scholars-Mexico Institute/El Colegio de la Frontera Norte.

París Pombo, M. D., Buenrostro Mercado, D. \& Pérez Duperou, G. (2017). Trapped at the border: the difficult integration of veterans, families, and christians in Tijuana. En B. Roberts, C. Menjívar \& N. P. Rodríguez (Eds.), Deportation and return in a border-restricted world: Experiences in Mexico, El Salvador, Guatemala and Honduras (pp. 131-148). Springer International Publishing. https://doi.org/10.1007/9783-319-49778-5_7

París, M. D., Hualde, A., \& Woo, O. (Coords.). (2019). Experiencias de retorno de migrantes mexicanos en contextos urbanos. El Colegio de la Frontera Norte. 
Pérez, G. (2014). La recomposición de las redes sociales de hombres expulsados por Estados Unidos a Tijuana, Baja California (tesis de maestría). El Colegio de la Frontera Norte. https://www.colef.mx/posgrado/wp-content/uploads/2014/11/TESIS-Perez-Duperou-Gabriel-Humberto-1.pdf

Pinillos, G. (2018). Volver a ser mexicano: reciudadanización y deportación en la frontera México-Estados Unidos (tesis de doctorado). El Colegio de la Frontera Norte. https://www.colef.mx/posgrado/wp-content/uploads/2018/10/TESIS-Pinillos-Quintero-Gabriela-Irina.pdf

Rivera, L. (2013). Migración de retorno y experiencias de reinserción en la zona metropolitana de la Ciudad de México. REMHU: Revista Interdisciplinar da Mobilidade Humana, 21(41), 55-76. https://doi.org/10.1590/S1980-85852013000200004

Sassen, S. (2013). Inmigrantes y ciudadanos: De las migraciones masivas a la Europa fortaleza. Siglo XXI.

Sayad, A. (2011). La doble ausencia: De las ilusiones del emigrado a los padecimientos del inmigrado. Anthropos.

Schuster, L. \& Majidi, N. (2013). What happens post-deportation? The experience of deported Afghans. Migration Studies, 1(2), 221-240. https://doi.org/10.1093/migration/mns011

Tilly, C. (1978). Migration in modern European History. En W. H. McNeill \& R. Adams (Eds.), Human migration: Patterns and Policies (pp. 48-72). Indiana University Press.

Torpey, J. (1998). Coming and going: on the state monopolization of the legitimate “means of movement". Sociological Theory, 16(3), 239-259.

Velasco, L. \& Coubès, M.-L. (2013). Reporte sobre dimensión, caracterización y áreas de atención a mexicanos deportados desde Estados Unidos. El Colegio de la Frontera Norte.

Velasco, L., París, M. D., Albicker, S., Pérez, G. \& Félix, C. (2017). Emergencia migratoria y solidaridad de la sociedad civil en Tijuana, 2016-2017. El Colegio de la Frontera Norte.

Velázquez de la Parra, M. (1991). El derecho a la vivienda. Cuadernos del Instituto de Investigaciones Jurídicas. Aspectos jurídicos de la vivienda, 6(18), 477-489. https://biblio. juridicas.unam.mx/bjv/id/1766

xxII Ayuntamiento Tijuana. (2017). Bando de policía y gobierno para el municipio de Tijuana, Baja California. https://web.archive.org/web/20180516205444/http://www. tijuana.gob.mx/reglamentos/Municipales/RM_BandodePoliciayGobierno_TJBC_11122017.pdf

Zelinsky, W. (1971). The hypothesis of the mobility transition. Geographical Review, 61(2), 219-249. https://doi.org/10.2307/213996

Gabriela Pinillos

Colombiana. Doctora en Ciencias Sociales con especialidad en Desarrollo Regional, en el Colegio de la Frontera Norte. Investigadora posdoctoral Seminario Migración, Desigualdad y Políticas Públicas. El Colegio de México. Ciudad de México, México. Líneas de especialidad: Desigualdades y procesos de construcción de ciudadanía, poder y control del Estado, estudios de fronteras, deportaciones y desplazamientos forzados. Publicación reciente: Pinillos, G. \& Flores, F. (2020). Migración de retorno y separación familiar: un estudio de caso en la Ciudad de México. Revista Coyuntura Demográfica, número especial, (18), 97-101. 